\title{
Changing with the times - developing chassis for the future of transportation and customer mobility
}

Charles J. Klein, Dr. V. Held, Adam Opel AG

This manuscript was not available on completion of this publication.

Thank you for your understanding. 